\title{
Matrix elasticity in vitro controls muscle stem cell fate in vivo
}

\author{
Matthew Raab, Jae-Won Shin and Dennis E Discher*
}

\begin{abstract}
Almost every laboratory that grows mammalian cells today grows their cells on tissue culture plastic, which was introduced to cell culture decades ago based on properties such as inertness, transparency, and so forth. However, plastic is rigid and unlike the many soft tissues in the body. Polymer gel systems that mimic the softness of various tissues have been developed over the past decade to test and understand the effects of rigidity on cells such as muscle cells. One recent study even shows that muscle stem cells expand much better in vitro on muscle-mimetic gels and that such cells prove optimal for engraftment in muscle.
\end{abstract}

Solid tissue repair or regeneration with stem cells is a major challenge that is now motivating studies of many cell types with many methods. Therapies are typically expected to require expansion of autologous or allogeneic cells before transplantation into damaged or diseased tissue, but an ability to control self-renewal, differentiation, and expansion of isolated stem cells and their progenitors is probably crucial for any successful translation to humans. Soluble factors have been the usual approach in efforts to control stem cells, but Gilbert and colleagues show that even transplantation into mice will benefit from more attention to insoluble factors [1].

Tissue cells are adherent, which engages many signaling pathways. Indeed, adhesion extends into a cell beyond its membrane, with active engagement of the cytoskeleton: a cell constantly probes its microenvironment by physically pulling on extracellular matrix and adjacent cells. Such forces cause matrix deformations in proportion to matrix elasticity; that is, the tendency of your tissue to spring back after pinching or pulling it. At the cell level, the mechanics feed back and remodel a cell's cytoskeleton, impacting signaling pathways and cell fate. A number of

*Correspondence: discher@seas.upenn.edu

University of Pennsylvania, 129 Towne Bldg., Philadelphia, PA, USA 19104 recent papers have highlighted the importance of myosin contractility in the viability and fate of pluripotent embryonic stem cells [2-4]. The coupling of stem-cellgenerated forces to matrix elasticity also affects differentiation, with initial results for mesenchymal stem cells indicating that matrix elasticity directs lineage specification [5]. Hematopoietic stem cells cultured on soft, elastic substrates expand twofold to threefold whereas crosslink rigidification of the substrate abolishes this effect [6]. Matrix stiffness indeed varies between tissues, and ranges from extremely soft bone marrow and brain tissue to rigid calcified bone. Muscle is not too soft and not too stiff, as muscle needs to be sufficiently compliant to change length in contraction. Moreover, when there are defects in compliant proteins, such as dystrophin [7], muscular dystrophies arise - which motivates therapies such as stem cell transplantation.

At the microscale relevant to cells, the lateral elasticity of normal, flaccid skeletal muscle has been measured to have an elastic modulus of $\sim 12 \mathrm{kPa}$ [8], while rat cardiac tissue [9] and mouse cardiac tissue [10] are perhaps stiffer by up to twofold. Such elasticity is typical of many polyacrylamide gels widely used in protein separations. Gilbert and colleagues reproduced past measurements of the tibialis anterior skeletal muscle and then determined whether this level of stiffness affects the behavior of muscle stem cells (MuSCs) [1]. Hydrogels were engineered to have the same elasticity as muscle tissue, or else were softer or stiffer, with laminin basement membrane protein integrated into the gels. MuSCs were thus cultured on gels of elasticities $2 \mathrm{kPa}, 12 \mathrm{kPa}$, and $42 \mathrm{kPa}$, and also on extremely thin gels on plastic so that the cells could feel the rigid plastic beneath. Tissue culture plastic is about 100,000-fold more rigid than any soft tissue.

Surprisingly, MuSCs cultured on $12 \mathrm{kPa}$ gels expanded over time and maintained a primitive phenotype, whereas MuSCs on rigid substrates did not expand and also tended to differentiate. Time-lapse imaging showed that while the cells divided at similar rates on both soft and rigid substrates, cells on rigid substrates died much more frequently. Two transcription factors were assayed to stage the cells: Pax7 for MuSCs, and Myogenin for 


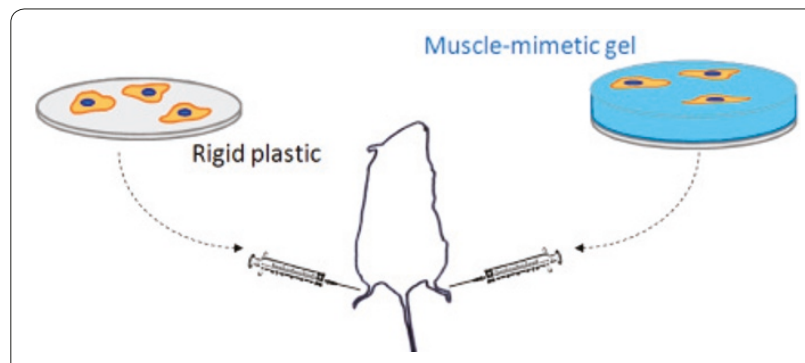

Figure 1. Priming cells on rigid plastic versus biomimetic gels.

differentiation. Pax7 was seen in about one-third of dividing cell doublets on the $12 \mathrm{kPa}$ matrices, indicating symmetric division and self-renewal; this was about fivefold higher than doublets on rigid substrate. Myogenin immunofluorescence also suggested a threefold lower expression on the $12 \mathrm{kPa}$ matrices, consistent with a less differentiated state. Past studies of mesenchymal stem cells on $\sim 12 \mathrm{kPa}$ showed that both Pax7 and Myogenin were induced but only to levels well below myoblasts [5]. Earlier work with myoblasts further demonstrated that myoblasts would fuse and generate the most robustly striated myotubes on $12 \mathrm{kPa}$ matrices compared with even $50 \%$ softer or $50 \%$ stiffer substrates [8]. Multiple in vitro studies have thus demonstrated that $12 \mathrm{kPa}$ matrices are best for multiple stages of myogenesis.

Transplantation of matrix-controlled cells was therefore the next logical step for the field. Gilbert and colleagues expanded MuSCs on $12 \mathrm{kPa}$ gels for 7 days and then injected them into damaged muscle (Figure 1). While freshly isolated MuSCs that are immediately transplanted into injured muscle tissue engraft best, even one cycle of division on rigid plastic prevents any significant MuSC engraftment. In comparison, just $10 \mathrm{MuSCs}$ that had undergone one round of division on $12 \mathrm{kPa}$ gels proved sufficient for $10 \%$ above threshold engraftment upon transplantation. Growth of MuSCs in vitro for even a short time on muscle-mimetic matrix can thus promote self-renewal and prolong regenerative potential. Whether this material approach to stem cell expansion will apply to other progenitor cells needs to be tested. Also in need of testing for more lineages than muscle is whether matrices can be used to first direct in vitro differentiation of more pluripotent or multipotent stem cells and then expand the early progenitors as a method to fully prime for implantation. It is nonetheless becoming clear that stem cells feel matrix elasticity as a potent insoluble factor in proliferation and differentiation.

\section{Abbreviations}

MuSC, muscle stem cell.

\section{Competing interests}

The authors declare that they have no competing interests.

\section{Published: 10 December 2010}

\section{References}

1. Gilbert PM, Havenstrite KL, Magnusson KE, Sacco A, Leonardi NA, Kraft P, Nguyen NK, Thrun S, Lutolf MP, Blau HM: Substrate elasticity regulates skeletal muscle stem cell self-renewal in culture. Science 2010, 329:1078-1081.

2. Walker A, Su H, Conti MA, Harb N, Adelstein RS, Sato N: Non-muscle myosin II regulates survival threshold of pluripotent stem cells. Nat Commun 2010, $1(6)$

3. Ohgushi M, Matsumura M, Eiraku M, Murakami K, Aramaki T, Nishiyama A, Muguruma K, Nakano T, Suga H, Ueno M, Ishizaki T, Suemori H, Narumiya S, Niwa H, Sasai Y: Molecular pathway and cell state responsible for dissociation-induced apoptosis in human pluripotent stem cells. Cell Stem Cell 2010, 7:225-239.

4. Chen G, Hou Z, Gulbranson DR, Thomson JA: Actin-myosin contractility is responsible for the reduced viability of dissociated human embryonic stem cells. Cell Stem Cell 7:240-248.

5. Engler AJ, Sen S, Sweeney HL, Discher DE: Matrix elasticity directs stem cell lineage specification. Cell 2006, 126:677-689.

6. Holst J, Watson S, Lord MS, Eamegdool SS, Bax DV, Nivison-Smith LB, Kondyurin A, Ma L, Oberhauser AF, Weiss AS, Rasko JE: Substrate elasticity provides mechanical signals for the expansion of hemopoietic stem and progenitor cells. Nat Biotechnol 2010, 28:1123-1128.

7. Carag-Krieger C, Bhasin N, Tewari M, Brown AE, Safer D, Sweeney HL, Discher DE: Exon-skipped dystrophins for treatment of Duchenne Muscular Dystrophy: domain structures based on single molecule mechanics with cooperativity in forced unfolding as a key to design. Cytoskeleton 2010, 67:796-807.

8. Engler AJ, Griffin MA, Sen S, Bonnemann CG, Sweeney HL, Discher DE: Myotubes differentiate optimally on substrates with tissue-like stiffness: pathological implications for soft or stiff microenvironments. J Cell Biol 2004, 166:877-887.

9. Berry MF, Engler AJ, Woo YJ, Pirolli TJ, Bish LT, Jayasankar V, Morine KJ, Gardner TJ, Discher DE, Sweeney HL: Mesenchymal stem cell injection after myocardial infarction improves myocardial compliance. Am J Physiol Heart Circ Physiol 2006, 290:H2196-H2203.

10. Jacot JG, Martin JC, Hunt DL: Mechanobiology of cardiomyocyte development. J Biomech 2009, 43:93-98.

doi:10.1186/scrt38

Cite this article as: Raab M, et al:: Matrix elasticity in vitro controls muscle stem cell fate in vivo. Stem Cell Research \& Therapy 2010, 1:38. 